Conclusion The palliative care team were referred patients with Covid-19 at a later stage in their illness. The symptom burden and medication requirements at the end of life were however similar across all groups. It is helpful to be aware of these findings so that we can care for patients dying of Covid-19 effectively.

\section{DO PATIENTS WITH COVID-19 REQUIRE DIFFERENT END OF LIFE MEDICATIONS? - THE EXPERIENCES OF A DUAL SITE HOSPITAL PALLIATIVE CARE TEAM}

Lisa Boulstridge, Jillian Wall, Ruth England, Hannah Curtis. University Hospitals of Derby and Burton

\subsection{6/spcare-2021-PCC.37}

Introduction University Hospitals of Derby and Burton (UHDB) report our Hospital Palliative Care Team's (HPCT) experience in supporting patients with COVID-19 during the pandemic's first surge.

Methods Inclusion criteria: patients supported by HPCT at two acute hospital sites, with a positive RT-PCR nasopharyngeal swab for SARS-CoV-2 between 16th March and 1st May 2020. Exclusion criteria: Patients intubated on ITU. Data was extracted from medical and nursing notes retrospectively and prescriptions in the last 3 days of life were reviewed. We sought to describe the cohort of COVID-19 patients supported by HPCT and to evaluate their medication requirements at end of life.

Results 223 patients were referred to HPCT: 155 (70\%) with a positive swab for SARS-CoV-2. 95\% had never been seen by HPCT previously. On average they had had one hospital admission in the preceding 12 months (range 1-8). The proportion of non-white patients was higher than our usual cohort (7 vs 4.6\%). Whilst receiving input from HPCT 112 $(72 \%)$ patients died in hospital and only $4 \%$ were discharged from hospital. All patients had anticipatory medications prescribed during their last 3 days of life; $41 \%$ required at least one dose in the 2 days before death, and $62 \%$ on the day of death. On the day of death, 59\% required a dose of an opiate, $60 \%$ midazolam and $20 \%$ an antipsychotic; $72 \%$ had a syringe driver in place. However $23 \%$ required no anticipatory medications or a syringe driver. Doses of drugs given did not exceed those typically used in non-COVID patients.

Conclusions The vast majority of patients during this period of time were new referrals to HPCT. In line with other published work, most patients dying with COVID-19 had symptoms which were managed with usual doses of medications. An opiate and midazolam were the drugs most frequently required.

\section{DEVELOPING SYMPTOM CONTROL GUIDANCE FOR COVID-19 PATIENTS WHO ARE DETERIORATING DESPITE NON-INVASIVE VENTILATION (NIV) AS THEIR CEILING OF TREATMENT}

Lowri Evans, Clea Atkinson, Anthony Byrne. Cardiff and Vale University Health Board and Velindre University NHS Trust, Cardiff

10.1136/spcare-2021-PCC.38

Background COVID-19 positive patients often develop Type 1 respiratory failure requiring $\mathrm{CPAP}$, meaning deteriorating patients are often alert, leading to a challenging end of life situation at the time of NIV discontinuation. The Palliative Care team were asked to help better manage symptoms for COVID-19 positive patients approaching the end of life, and specifically those patients having NIV support as their ceiling of treatment, who were deteriorating very rapidly despite this. Methods A case note review was performed. Guidance was developed following rapid review of existing published guidance for NIV withdrawal and sedation for other comparable clinical situations, with adaptation using the experiential knowledge of the palliative care and respiratory teams in managing COVID-19 positive patients. Finally, to evaluate the guideline an online survey was distributed to respiratory ward staff following the 'first wave'.

Results A symptom control flowchart was developed and agreed for use in the health board specifically for managing the rapidly deteriorating patient with COVID-19 on NIV as their ceiling of treatment. This later formed part of the All Wales COVID-19 National Secondary Care Guidance website. The online survey drew 12 respondents. Following introduction of the guideline the proportion of respondents who felt symptoms were adequately controlled rose from $17 \%$ to $58 \%$. Qualitative feedback demonstrated that despite the difficult circumstances, patients were treated with compassion and overall better symptom control was achieved. 91\% of relevant staff felt comfortable with the medication doses recommended for symptom control.

Conclusions Key to the appropriate implementation of this guidance has been the support of the local palliative care team and a good working relationship with the respiratory teams. A collaborative approach facilitated the rapid development of guidance in response to a direct patient need. This flowchart has given the respiratory team guidance to support better end of life care and reduce distress for patients, families and staff.

\section{THE EFFECT OF THE COVID 19 PANDEMIC ON REFERRALS AND DEATHS AT MEADOW HOUSE HOSPICE (MHH)}

Lucy Ison, Treena Saini. Meadow House Hospice

10.1136/spcare-2021-PCC.39

Background COVID 19 has caused untold levels of suffering throughout the world. Palliative care is essential, however access may be limited due to demand (Radbruch, et al., 2020). Services have had to respond with flexibility and rapid changes to systems and policies (Etkind, et al., 2020). In light of this we looked at data on referrals to see the impact that COVID 19 has had at MHH.

Methods We compared data on referrals and deaths from January to June 2020 with data from previous years.

Results Referrals to Meadow House hospice were steady during the first half of 2020 compared to previous years, with 1095 referrals from January - June 2020 compared to 1934 from January - December 2019. There were more community referrals with 970 referrals from January - June compared to 1618 in the whole of 2019. Lymphoedema referrals in contrast were reduced with 57 referrals from January - June 2020 compared to 170 from January - December 2019. There was a peak of referrals in April with 186 referrals in 2020 compared to 119 referrals in April 2019. 